\title{
Determinación del biotipo facial según el índice VERT de Ricketts y el ángulo de apertura facial
}

\author{
Determination of the Facial Biotype According to the Vert Ricketts Index and the Facial \\ Opening Angle
}

Wilber Dante Calla Enriquez

(1) https://orcid.org/0000-0002-2703-9584

\section{Resumen}

Objetivo: Determinar el biotipo facial según el índice VERT de Ricketts y el ángulo de apertura facial en pacientes de la Clínica Odontológica de la Universidad Nacional Jorge Basadre Grohmann en el año 2017. Material y métodos: Estudio de tipo no experimental, descriptivo, comparativo y transversal. La muestra estuvo conformada por 94 pacientes (38 hombres y 56 mujeres). Se utilizó el índice VERT de Ricketts para registrar y evaluar los cinco valores cefalométricos que determinan el biotipo facial a través de tejidos blandos y en una fotografía facial en norma frontal estandarizada para valorar el ángulo de apertura facial. Resultados: La aplicación del coeficiente de Kappa ponderado para evaluar la concordancia diagnóstica entre ambos análisis, reveló ausencia de acuerdo o concordancia $(k=-0,61)$ entre el índice VERT de Ricketts y el ángulo de apertura facial. También se observó ausencia respecto al sexo masculino $(k=-0,197)$ y femenino $(k=-$ $0,033)$. Conclusiones: No existe concordancia diagnóstica del biotipo facial entre el índice VERT de Ricketts y el ángulo de apertura facial $(p>0,05)$, tanto en relación al sexo masculino como al femenino $(p>0,05)$.

\section{Palabras clave: biotipo facial, cefalometría, clínico, comparativo}

\section{Abstract}

Objective: To determine the facial biotype according to the Ricketts VERT index and the facial opening angle in patients of the Dental Clinic of the Jorge Basadre Grohmann National University in 2017. Material and methods: Non-experimental, descriptive, comparative and cross. The sample consisted of 94 patients ( 38 men and 56 women). The Ricketts VERT index was used to record and evaluate the five cephalometric values that determine facial biotype through soft tissues and in a standardized frontal norm facial photograph to assess the facial opening angle. Results: The application of the weighted Kappa coefficient to evaluate the diagnostic agreement between both analyzes revealed lack of agreement or agreement $(\mathrm{k}=-0.61)$ between the Ricketts VERT index and the facial opening angle. Absence was also observed with respect to male $(k=-0.197)$ and female $(k=-0.033)$ sex. Conclusions: There is no diagnostic concordance of the facial biotype between the Ricketts VERT index and the facial opening angle $(p>0.05)$, both in relation to the male and female sex $(p>0.05)$.

Keywords: facial biotype, cephalometry, clinical, comparative 


\section{Introducción}

En la literatura ortodóntica encontramos que el biotipo facial puede ser determinado por medio de análisis en radiografía lateral de cráneo o por diferentes métodos de análisis facial de tejidos blandos. ${ }^{1,2}$

La dirección, magnitud del crecimiento y desarrollo cráneo facial son aspectos fundamentales para el análisis del botipo facial, sea este análisis cefalómetro o clínico. Tales aspectos otorgan luz clara para aplicar la biomecánica correspondiente y poder solucionar la maloclusión.

El método cefalométrico más utilizado en la determinación del biotipo facial es el índice VERT de Ricketts. ${ }^{3}$ El autor introduce el concepto de biotipo facial, el cual es definido como el conjunto de caracteres morfológicos y funcionales que determinan la dirección de crecimiento y comportamiento de la cara. En cambio, el ángulo de apertura facial es el ángulo que se forma por la intersección de dos líneas que van desde el punto más lateral de la órbita (punto cantal externo) hasta las comisuras labiales (punto cheilon), tanto del lado derecho como del lado izquierdo. Este ángulo debe tener un valor promedio de $45^{\circ}$ con una desviación estándar de $+/-5$. Los valores mayores a este ángulo indican una cara más ancha y cuadrada y valores menores indican una cara larga y estrecha. ${ }^{4}$

En la actualidad hay un creciente interés por el análisis facial para la elaboración del diagnóstico y planificación del tratamiento ortodóntico. Es fundamental conocer la interrelación entre el tejido tegumentario facial y la estructura esquelética subyacente y determinar el biotipo facial en cada individuo, de tal forma que el tratamiento ortodóntico pueda proporcionar un mejor equilibrio facial y una mayor estabilidad del resultado.

La utilización de fotografías estandarizadas en el diagnóstico ortodóntico es frecuente, puesto que retrata la cara de un individuo; además, la determinación del biotipo facial por medio del ángulo de apertura facial puede ser evaluada correctamente por el método fotográfico. Por lo tanto, es fundamental que estas fotografías sean de buena calidad y estandarizadas. ${ }^{5}$ El objetivo del presente estudio fue determinar el biotipo facial según el índice VERT de Ricketts y el ángulo de apertura facial en pacientes que acudieron a la Clínica Odontológica de la Universidad Nacional Jorge Basadre Grohmann en el año 2017. Tales resultados permiten comparar con precisión el diagnóstico del biotipo facial a través del análisis de tejido blando y duro, así como proveer información para asistir en el diagnóstico y planificación del tratamiento ortodóntico.

\section{Material y métodos}

La muestra estuvo conformada por 94 pacientes (38 hombres y 56 mujeres), cuyas edades oscilaron entre 9 a 18 años y fue determinado por conveniencia. Se utilizó el índice VERT de Ricketts para registrar y evaluar los cinco valores cefalométricos que determinan el biotipo facial. Para valorar el patrón facial a través de los tejidos blandos, se utilizó el ángulo de apertura facial, cuyos valores se obtuvieron en una fotografía facial en norma frontal estandarizada de tamaño 10 $x 15 \mathrm{~cm}$. Primero, los pacientes fueron ubicados a $30 \mathrm{~cm}$ delante de un fondo de pantalla con ambos brazos libres a un lado del tronco, con los pies separados para mayor estabilidad y con la cabeza en posición natural. Cada paciente fue instruido a mantener esa postura hasta la toma fotográfica. Se utilizó una cámara fotográfica compacta marca Canon modelo Power Shot SX 100 IS, montado en un trípode ubicado a $150 \mathrm{~cm}$ del paciente. Luego de obtener la impresión fotográfica en papel tamaño A4, se realizó el trazado de los puntos fotométricos, tales como Exocanto (Ex): Punto más lateral de la órbita, tanto derecho e izquierdo y Queilon (Ch): Punto más lateral de la boca, en la unión del labio superior e inferior (comisuras). Posteriormente, se unió dichos puntos y se obtuvo dos líneas fotométricas oblicuas. La primera de ellas, Línea $\mathrm{Ex}_{\mathrm{d}}-\mathrm{ch}_{\mathrm{d}}$ : línea oblicua que une los puntos exocanto derecho y queilon derecho; la segunda, línea $E x_{i}-c_{i}$ : línea oblicua que une los puntos exocanto izquierdo y queilon izquierdo. Así, la intersección de dichas líneas da un ángulo cuyo valor promedio es de $45^{\circ}$ y una 
desviación estándar de $+/-5^{\circ}$, lo cual indica una cara normal; ya que, un valor mayor a $50^{\circ}$ indica una cara cuadrada y un valor menor a $40^{\circ}$ indica una cara larga. ${ }^{4}$

\section{Análisis estadístico}

La información se almacenó en una base de datos automatizada en el programa Microsoft Office Excel 2003 sobre Windows XP profesional. Para el procesamiento de los resultados se utilizó el programa SPSS versión 18 y Microsoft Excel. El cálculo del error de método fue estimado con el Coeficiente de Correlación Intraclase $(\mathrm{CCl})$ y los resultados se presentan en tabla de frecuencia y con el valor estimado de la prueba estadística del coeficiente kappa ponderado (k). Para el contraste de la hipótesis, se recurrió a la prueba estadística de contraste de correlación de rho de Spearman.

\section{Resultados}

En la Tabla 1 se observa un coeficiente kappa ponderado con un valor negativo $(\mathrm{k}=-0,61)$ y con un intervalo de confianza que pasa por cero, lo que indica ausencia de concordancia entre el índice VERT de Ricketts y el ángulo de apertura facial.

En el análisis de datos sobre la concordancia del tipo de cara por medio de la cefalometría y el análisis facial, 43 pacientes presentaron concordancia en la categoría del biotipo dolicofacial y de cara larga tanto en el análisis cefalométrico como en el fotométrico. Tal cifra corresponde aproximadamente a casi la mitad de la muestra $(45,7 \%)$. De igual forma, casi la mitad de pacientes mesofaciales fueron clasificados como de cara normal $(n=29 ; 30,9 \%)$.

En cuanto a la tipología del patrón cara cuadrada, el ángulo de apertura facial y el biotipo facial braquifacial según el índice VERT de Ricketts; no se encontró concordancia entre tales indicadores respecto a sus categorías correspondientes. Asimismo, los 22 braquifaciales $(23,4 \%)$ fueron clasificados como cara normal.
De acuerdo a los resultados obtenidos en la Tabla 2, se aprecia un coeficiente kappa ponderado con un valor negativo y con un intervalo de confianza que pasa por cero. Esto indica ausencia de concordancia entre el coeficiente de variación vertical (VERT) de Ricketts y las medidas angulares de apertura facial en los hombres $(k=-0,197)$.

También, se observó un 42,1\% de hombres $(n=16)$ que presentó concordancia respecto al patrón facial dolicofacial y el tipo de cara larga tanto para el análisis facial fotométrico como para el cefalométrico. Asimismo, se observó que el $34,2 \%$ de hombres mesofaciales fue clasificado como cara normal. No hubo concordancia entre pacientes con cara cuadrada según el análisis del ángulo de la apertura facial y el biotipo facial braquifacial según el coeficiente de variación vertical de Ricketts. Por lo tanto, se identificó a 9 hombres braquifaciales $(23,7$ $\%$ ) que fueron clasificados como cara normal.

De acuerdo a los resultados obtenidos en la Tabla 2, se aprecia un coeficiente kappa ponderado con un valor negativo y con un intervalo de confianza que pasa por cero, lo que indica ausencia de concordancia entre el coeficiente de variación vertical (VERT) de Ricketts y las medidas angulares de apertura facial en las mujeres $(k=-0,033)$.

También, el $48,2 \%$ de mujeres $(n=27)$ presentó concordancia respecto al patrón facial dolicofacial y el tipo de cara larga, correspondientes para ambos análisis facial fotométrico y cefalométrico.

Respecto al sexo femenino, se observó que el 25,6 \% de mujeres mesofaciales fue clasificado como cara normal. No se observó concordancia entre pacientes con cara cuadrada (según el análisis del ángulo de la apertura facial) y el biotipo facial braquifacial (según el coeficiente de variación vertical de Ricketts) clasificado como cara normal. Por tanto, 13 mujeres braquifaciales $(23,2 \%)$ fueron clasificados como cara normal. 


\section{Tabla 1}

Concordancia diagnóstica del biotipo facial según índice VERT de Ricketts y el ángulo de apertura facial

\begin{tabular}{|c|c|c|c|c|c|c|c|c|c|c|c|}
\hline \multirow[t]{2}{*}{ Método } & \multicolumn{6}{|c|}{ Angulo de apertura facial } & & & \multirow{3}{*}{$\begin{array}{l}\text { Kappa } \\
\text { ponderado }\end{array}$} & \multirow{2}{*}{\multicolumn{2}{|c|}{$\begin{array}{lc}\text { Interva } & \text { de } \\
\text { confianza } & (95 \%) \\
\end{array}$}} \\
\hline & \multicolumn{2}{|c|}{$\begin{array}{l}\text { Cara } \\
\text { larga }\end{array}$} & \multicolumn{2}{|c|}{$\begin{array}{l}\text { Cara } \\
\text { normal }\end{array}$} & \multicolumn{2}{|c|}{$\begin{array}{l}\text { Cara } \\
\text { cuadrada }\end{array}$} & \multicolumn{2}{|c|}{ Total } & & & \\
\hline Índice VERT & $\mathrm{n}$ & $\%$ & $\mathrm{n}$ & $\%$ & $\mathrm{n}$ & $\%$ & $\mathrm{n}$ & $\%$ & & Inferior & Superior \\
\hline $\begin{array}{l}\text { Dolicofacial } \\
\text { severo }\end{array}$ & 9 & 9,6 & 0 & 0,0 & 0 & 0,0 & 9 & 9,6 & $-0,61$ & $-1,27$ & 0,007 \\
\hline Dolicofacial & 18 & 19,1 & 0 & 0,0 & 0 & 0,0 & 18 & 19,1 & & & \\
\hline $\begin{array}{l}\text { Dolicofacial } \\
\text { leve }\end{array}$ & 16 & 17,0 & 0 & 0,0 & 0 & 0,0 & 16 & 17,0 & & & \\
\hline Mesofacial & 0 & 0,0 & 29 & 30,9 & 0 & 0,0 & 29 & 30,9 & & & \\
\hline Braquifacial & 0 & 0,0 & 16 & 17,0 & 0 & 0,0 & 16 & 17,0 & & & \\
\hline $\begin{array}{l}\text { Braquifacial } \\
\text { severo }\end{array}$ & 0 & 0,0 & 6 & 6,4 & 0 & 0,0 & 6 & 6,4 & & & \\
\hline Total & 43 & 45,7 & 51 & 54,3 & 0 & 0,0 & 94 & 100 & & & \\
\hline
\end{tabular}

Fuente: Ficha de registro de datos. Prueba: k.

\section{Tabla 2}

Concordancia diagnóstica del biotipo facial entre el índice VERT de Ricketts y el ángulo de apertura facial

\begin{tabular}{|c|c|c|c|c|c|c|c|c|c|c|c|c|}
\hline \multirow[t]{3}{*}{ Género } & \multirow{3}{*}{$\begin{array}{l}\text { Método } \\
\text { Indice Vert }\end{array}$} & \multicolumn{7}{|c|}{ Angulo de apertura facial } & \multirow[b]{2}{*}{ Total } & \multirow{3}{*}{$\begin{array}{l}\text { Kappa } \\
\text { ponderado }\end{array}$} & \multirow{2}{*}{\multicolumn{2}{|c|}{$\begin{array}{lr}\text { Intervalo } & \text { de } \\
\text { confianza }(95 \%)\end{array}$}} \\
\hline & & \multicolumn{2}{|c|}{$\begin{array}{l}\text { Cara } \\
\text { larga }\end{array}$} & \multicolumn{2}{|c|}{$\begin{array}{l}\text { Cara } \\
\text { normal }\end{array}$} & \multicolumn{3}{|c|}{$\begin{array}{l}\text { Cara } \\
\text { cuadrada }\end{array}$} & & & & \\
\hline & & $\mathrm{n}$ & $\%$ & $\mathrm{n}$ & $\%$ & $\mathrm{n}$ & $\%$ & $\mathrm{n}$ & $\%$ & & Inferior & Superior \\
\hline \multirow[t]{7}{*}{ Masculino } & $\begin{array}{l}\text { Dolicofacial } \\
\text { severo }\end{array}$ & 2 & 5,3 & 0 & 0,0 & 0 & 0,0 & 2 & 5,3 & $-0,107$ & $-0,201$ & 0,32 \\
\hline & Dolicofacial & 8 & 21,1 & 0 & 0,0 & 0 & 0,0 & 8 & 21,1 & & & \\
\hline & $\begin{array}{l}\text { Dolicofacial } \\
\text { leve }\end{array}$ & 6 & 15,7 & 0 & 0,0 & 0 & 0.0 & 6 & 15,7 & & & \\
\hline & Mesofacial & 0 & 0,0 & 13 & 34,2 & 0 & 0,0 & 13 & 34,2 & & & \\
\hline & Braquifacial & 0 & 0,0 & 6 & 15,8 & 0 & 0,0 & 6 & 15,8 & & & \\
\hline & $\begin{array}{l}\text { Braquifacial } \\
\text { severo }\end{array}$ & 0 & 0,0 & 3 & 7,9 & 0 & 0,0 & 3 & 7,9 & & & \\
\hline & Total & 16 & 42,1 & 22 & 57,9 & 0 & 0,0 & 38 & 100 & & & \\
\hline \multirow[t]{7}{*}{ Femenino } & $\begin{array}{l}\text { Dolicofacial } \\
\text { severo }\end{array}$ & 7 & 12,5 & 0 & 0,0 & 0 & 0,0 & 7 & 12,5 & $-0,033$ & $-0,119$ & 0,032 \\
\hline & Dolicofacial & 10 & 17,8 & 0 & 0,0 & 0 & 0,0 & 10 & 17,8 & & & \\
\hline & $\begin{array}{l}\text { Dolicofacial } \\
\text { leve }\end{array}$ & 10 & 17,9 & 0 & 0.0 & 0 & 0,0 & 10 & 17,9 & & & \\
\hline & Mesofacial & 0 & 0,0 & 16 & 25,6 & 0 & 0,0 & 16 & 25,6 & & & \\
\hline & Braquifacial & 0 & 0,0 & 10 & 17,8 & 0 & 0,0 & 10 & 17,8 & & & \\
\hline & $\begin{array}{l}\text { Braquifacial } \\
\text { severo }\end{array}$ & 0 & 0.0 & 3 & 5,4 & 0 & 0,0 & 3 & 5,4 & & & \\
\hline & Total & 27 & 48,2 & 29 & 51,8 & 0 & 0,0 & 56 & 100 & & & \\
\hline
\end{tabular}

Fuente: Ficha de registro de datos prueba. 


\section{Discusión}

Los resultados del presente estudio revelan información importante para una mejor comprensión en el diagnóstico del biotipo facial en la práctica ortodóntica y están basado en los resultados encontrados en los pacientes que acudieron a la Clínica Odontológica de la UNJBG. Sobre la concordancia diagnóstica del biotipo facial entre Índice VERT de Ricketts y el ángulo de apertura facial, se observa ausencia de concordancia $(k=-0,61)$ y de acuerdo a Banerjee et al. ${ }^{6}$, una débil concordancia (< $0,40)$.

Resultados similares fueron observados por Ramires et al., ${ }^{7}$ al determinar el biotipo en 105 brasileños leucodermas entre 20 y 40 años, utilizando el índice VERT de Ricketts y el análisis facial subjetivo. En este estudio, no se encontró correlación entre ambos análisis. También, nuestros resultados se asemejan con los de Curioca et al. ${ }^{8}$ Los autores evaluaron el biotipo facial en 89 niños de 6 a 10 años, empleando el índice facial morfológico y el índice VERT de Ricketts, y encontraron correlación entre ambos diagnósticos. De igual manera, Roco ${ }^{9}$ estudió la asociación entre el análisis fotográfico frontal y el análisis cefalométrico en 61 adultos jóvenes chilenos. En la determinación del biotipo facial, el autor no encontró correlación significativa $(r=0,133)$ entre el índice VERT de Ricketts y el ángulo del biotipo facial.

En cuanto a la coincidencia del tipo de cara entre el índice VERT de Ricketts y el análisis del ángulo de apertura facial, los resultados permitieron observar que 72 de los casos coincidieron y 22 no lo hicieron. De los casos que coincidieron $(76,6 \%)$, la mayoría de ellos eran pacientes con patrón dolicofacial $(n=43)$ y el resto mesofaciales ( $n$ =29), independientemente del sexo y edad. Además, no hubo coincidencia entre los braquifaciales con el tipo de cara cuadrada.

Duarte $^{10}$ describió la existencia de pacientes limítrofes entre los biotipo faciales; por ejemplo, casos clínicos donde el autor observó pacientes mesofaciales con tendencia suave a braquifacial (VERT $+0,52$ ). De igual forma, Martins y Vigorito ${ }^{11}$ reportaron casos donde los valores del índice VERT eran limítrofes entre dos tipos faciales y que los tejidos blandos pudieron enmascarar las características del tejido óseo subyacente. En lo referente a este último punto, en el trabajo longitudinal de Blanchette et al. ${ }^{12}$, se observó el crecimiento y desarrollo de los tejidos blandos en individuos desde los 7 hasta los 17 años. Sumado a esto, por medio de la telerradiografía de perfil, se observó que los individuos con patrón vertical cara corta presentaban menor espesor de los tejidos blandos, con excepción a nivel del punto $A$, en comparación a los de cara larga.

También, en el trabajo denominado Cambios longitudinales en tres tipos faciales normales de Bishara y Jakonbsen ${ }^{13}$, se reportó que la mayoría de los sujetos $(77 \%)$ tenía el mismo tipo de cara a los 5 años y 25,5 años de edad; por tanto, existía una fuerte tendencia a mantener el tipo facial original con la edad. Además, los sujetos en cada tipo facial tenían variaciones relativamente grandes en el tamaño y relaciones de las diversas estructuras dentofaciales. En el $23 \%$ de los sujetos restantes de la muestra hubo un cambio en la categorización del tipo facial entre 5 años y 25 años de edad. Es posible especular que la mayoría de estos casos fueron "limítrofes" entre dos tipos faciales y que los factores epigenéticos inclinaron el equilibrio de un tipo para el otro.

Las edades de los pacientes de nuestro estudio con patrón facial braquifacial oscilaron entre los 9 a 18 años y con un $50 \%$ de braquifaciales con un coeficiente de crecimiento vertical próximo a $+0,5$; y con características faciales menos acentuadas en relación a lo descrito por $\mathrm{Nanda}^{14}$, quien en su estudio longitudinal en participantes desde los 4 a los 18 años evidenció una tendencia de disminución de los ángulos formados por los planos horizontales de la cara. Este hecho agravaría las relaciones faciales de individuos de cara corta y manifestándose más con la mayoría de edad. Se puede destacar en nuestro estudio que no se observó ningún paciente con las cinco medidas angulares del índice VERT de Ricketts correspondientes para cada biotipo facial en la muestra estudiada. Contrariamente, existen medidas que se combinan, tal como lo reportado en el trabajo sobre crecimiento y desarrollo cráneo facial 
de Bjork ${ }^{15}$. En este último trabajo, el autor menciona la existencia de biotipos faciales en las cuales hay una mezcla de proporción más o menos iguales de los tres biotipos. ${ }^{15}$ Investigaciones sobre cambios durante el crecimiento y desarrollo cráneo facial, como el de Baughan et al. ${ }^{16}$, donde se estudió el patrón de crecimiento facial en una muestra étnica homogénea de 50 niños canadienses, antes y durante la pubertad, se observó que el crecimiento y desarrollo de la cara es menos pronunciado que el de la estatura durante el pico de crecimiento puberal. También, en otro estudio longitudinal, Stahl et al. ${ }^{17}$ observó los cambios durante el crecimiento y desarrollo cráneo facial durante todo el periodo circumpuberal en una muestra de 17 individuos con oclusión normal y 17 individuos con maloclusión clase ll división 1 y con diferente biotipo facial, donde el crecimiento cráneo facial era similar en ambos grupos, pero con la excepción de un incremento menor significativo en la longitud mandibular en el grupo de maloclusión clase II. Los autores concluyeron que las características anatómicas son mantenidas durante el periodo circumpuberal (pre puberal, puberal y post puberal), indicando que no hay corrección pasiva de la desarmonía dento-esqueletal, aún durante el pico de crecimiento puberal. Por otro lado, en el estudio biotipológico facial longitudinal de Conde et al. $^{18}$, realizado en una población cubana de 63 niños de 7 a 11 años, pertenecientes a dos etnias: europoides (europeos de origen mediterráneo, como la española) y negroides (descendencia africana). El estudio utilizó el índice Vert de Ricketts y se observó que tanto los negroides como los europoides mantenían un patrón biotipológico muy parecido desde los 7 hasta los 11 años. Sumado a esto, los europoides presentaron un patrón braquifacial bien definido; mientras que los negroides presentaron cierta tendencia al patrón mesofacial (Vert +0,31).

Los cambios en el crecimiento y desarrollo cráneo facial han sido investigados por Lande ${ }^{19}$, Inouye ${ }^{20}$ y Subtenly ${ }^{21}$. Estos autores han observado que el crecimiento facial raramente ocurre en forma constante, balanceada e igual en cantidad y/o dirección.
En relación a la coincidencia del tipo de cara entre el índice de VERT de Ricketts y el análisis del ángulo de apertura facial, se observó que la mayoría de hombres con patrón dolicofacial $(42,1 \%)$ fueron clasificados como de cara larga. Los individuos masculinos mesofaciales $(34,2 \%)$ fueron categorizados como cara normal y los hombres braquifaciales $(23,7 \%)$ también fueron considerados como cara normal.

En consideración al sexo femenino, se observó que el 48,2 \% de mujeres con patrón dolicofacial, fue considerado en la tipología de cara larga; en cambio las mujeres mesofaciales $(25,6 \%)$ fueron clasificadas en el tipo de cara normal. En relación a las mujeres braquifaciales $(23,2 \%)$, ellas fueron clasificadas como de tipo cara normal. Otros estudios reportaron resultados diferentes como el de Ramires et al. ${ }^{6}$ Los autores observaron que la mayoría de individuos masculinos con biotipo dolicofacial fueron clasificados en el tipo cara media $(n=4$; $22,6 \%$ ), los hombres mesofaciales fueron clasificados como cara media y corta. En el grupo de los hombres braquifaciales, la mayoría de ellos fueron considerados como de cara media y una minoría como cara corta. Asimismo, en el grupo de sexo femenino con patrón dolicofacial (55\%), la mayoría de ellas fueron categorizadas como cara media. De igual forma, la mayoría de las mujeres con patrón mesofacial $(n=16 ; 81,53 \%)$ fueron consideradas como cara media y en relación a las mujeres con patrón braquifacial en su mayoría fueron identificadas como cara corta y el resto cara media.

\section{Conclusiones}

- No hubo concordancia diagnóstica del biotipo facial entre el Índice VERT de Ricketts y el ángulo de apertura facial $(\mathrm{k}=\mathrm{-}$ 0,61).

- No se encontró concordancia diagnóstica en la determinación del biotipo facial entre el índice VERT de Ricketts y el ángulo de apertura facial en el sexo masculino $(\mathrm{k}=$ 0,197).

- No se encontró concordancia diagnóstica en la determinación del biotipo facial entre el índice VERT de Ricketts y el ángulo de apertura facial en el sexo femenino $(k=-$ $0,033)$. 


\section{Referencias}

1. Ricketts RM. Planning treatment on the basis of the facial pattern and an estimate of its growth. The Angle Orthod. 1957; 27(1):14-37. Doi: $\begin{array}{llllllllllll}1 & 0 & 1 & 0 & 4 & 3 & / & 0 & 0 & 0 & 3 & -\end{array}$ 3219(1957)027<0014:PTOTBO>2.0.C $0 ; 2$.

2. Scanavini C. Vigorito JW. Estudo cefalométrico-radiográfico das possíveis correlações existentes entre as análises de Vigorito, Ricketts e Siriwat \& Jarabak na definição dos tipos faciais, em individuos leucodermas. Ortodontia 2001;34(3):27-41. Disponible en: shorturl.at/choy3.

3. Ricketts RM, Roth RM, Chaconas SJ, Schulhof RJ. Gary A Engel Orthodontics diagnosis and planning.: Their Roles in Preventive and Rehabilitative Dentistry. Rocky Mountain/Orthodontics, Denver. 1982

4. Viazis AD. Avaliação do tecido mole. In: Viazis AD. Atlas de Ortodontia: princípios e aplicações clínicas. São Paulo: Ed. Santos, 1996. p. 49-57.

5. Scanavini MA, Trevisan F, Maltagliati LA, Santos JE, Martelli Filho JA. Novo dispositivo para obtenção de fotografias frontais e laterais padronizadas. J Bras Ortodont Ortop Facial. May-jun 2003; 8(45):245-50. Disponible en: shorturl.at/bPW89.

6. Banerjee M, Capozzoli M, McSweeney L, Sinha D. Beyond Kappa: a review of interrater agreemente measures. Can $\mathrm{J}$ Stat. 1999;27(1):3-23. Doi: $10.2307 / 3315487$.

7. Ramires RR, Ferreira LP, Marchesan IQ, Cattoni DM, Andrada e Silva MA. Relação entre cefalometria e análise facial na determinação do tipo de face. Rev. CEFAC. 2009, 11(3):349-354. Disponible en: shorturl.at/aolP9.

8. Curioca S, Portillo G. Determinación clínica y radiográfica del somatotipo facial en pacientes pediátricos Revista
Odontológica Mexicana 2011;15 (1): 813. D i s p o i b e e n : https://www.medigraphic.com/pdfs/odo n/uo-2011/uo111b.pdf.

9. Roco R. Determinación del biotipo facial mediante un análisis fotográfico frontal y su correlación con dos métodos cefalométricos [Tesis de pregrado]. Santiago, Chile: Universidad de Chile Facultad de Odontología; 2014. D i s p n i ble e n : http://repositorio.uchile.cl/handle/2250/ 131646

10. Duarte MS. Manual de Cefalometria de Ricketts. 2004.

11. Martins LF, Vigorito JW. Photometric analysis applied in determining facial type. Dental Press J Orthod. Set-oct 2012; 17(5): 71-5. Doi: 10.1590/S217694512012000500010.

12. Blanchette ME, Nanda RS, Currier GF, Ghosh J, Nanda SK. A longitudinal cephalometric study of the soft tissue profile of short- and long-face syndromes from 7 to 17 years. Am J Orthod Dentofacial Orthop. Feb $1996 ; 109$ (2): 116-31. Doi : 10.1016/s0889-5406(96)70172-5

13. Bishara SE, Jakobsen JR. Longitudinal changes in three normal facial types. Am J Orthod 1985; 88:466-502. Doi: 10.1016/s0002-9416(85)80046-4.

14. Nanda S. Growth patterns in subjects with long and short faces. Am J Orthod Dentofacial Orthop. Sept 1990; 98 (3): 247-58. Doi: 10.1016/S08895406(05)81602-6.

15. Björk A. The significance of growth changes in facial pattern and their relationship to changes in occlusion. Dent Rec. Nov 1951; 71(10):197-208. PMID: 14887399.

16. Baughan B, Demirjian A, Levesque GY, Lapalme-Chaput L. T. The pattern of facial growth before and during puberty, as show by French-Canadian girls. Annals of human biology, 1979, 6, (1): 
$59-76$.

Doi: $10.1080 / 03014467900003371$.

17. Stahl F, Baccetti T, Franchi L, Mcnamara JA. Longitudinal growth changes in untreated subjects with Class Division 1 malocclusion. Am J Orthod Dentofacial Orthop. Jul 2008; 34(1): 125-37. Doi: 10.1016/j.ajodo.2006.06.028.

18. Conde Suárez HF, Valentín González F, Gou Godoy MA. Estudio biotipológico facial longitudinal en dentición mixta, análisis por grupos étnicos. revmedicaelectronica [Internet]. 2014; 30(1): [aprox.6p.].Disponible en: http://www.revmedicaelectronica.sld.cu /index.php/rme/article/view/466.
19. Lande MJ: Growth behavior of the human body facial profile as revealed by serial cephalometric roentenology. Angle Orthod. 1952; 22:78-90.

20. Inouye SY: A serial study of the soft tissue profile of individuals with excellent occlusions [M.S.D. thesis]. University of Washington, 1957

21. Subtelny JD: A longitudinal study of soft tissue facial structures and their profile characteristics, defined in relation to underlying skeletal structures. Am J Orthod. Jul 1959; 45(7): 481-507. Doi.org/10.1016/0002-9416(59)90014-4. 